Ophthalmic Experiences in the Royal Air Force 601

\title{
OPHTHALMIC EXPERIENCES IN THE ROYAL AIR FORCE
}

\author{
BY
}

FRANK R. NEUBERT

LONDON

DURING the Great War, 1914-18, it was estimated that, due to physical defects, the service of over one million men was lost to the British armed forces.

During the present war there has been a second opportunity to examine the physical condition of a large section of the population and assess the prevalence of certain conditions which normally do not bring the "sufferer" before a doctor. The subjects on whom this paper is written were invariably unaware of the peculiarities, which were discovered during the course of routine examinations.

'The records cover the period from June 1941 until August 1945.

No claim is made for statistical accuracy, and the value of the figures for scientific purposes is small. Records could not always be kept, and as many of the candidates had been examined previously some interesting cases must have been rejected.

The exceptionally good ophthalmic condition of the men from Holland and Norway is noteworthy. Only one rolunteer was seen with defective colour vision (from Holland) but it is not possible to say how well these men had been examined previously.

The manner in which the test types were read by emmetropes varied so much that one wondered whether an attempt had ever been made to make a comparison between the number of retinal elements and cortical cells: One man would read the $6 / 4$ line as rapidly as he could enunciate the letters, while another would stumble slowly but correctly down the whole chart, eventually to read the same line. It is unfortunate that the pressure of work made it impossible to check these responses with the man's intelligence quotient.

Criticism has been made of the different visual acuities recorded at different examinations, but as the conditions are variable these differences must be expected.

\section{Visual acuity}

A visual acuity of $6 / 6$, is an average figure which is usually accepted as the normal, but it was so common to find pilots originally passed as $6 / 6,6 / 6$, who could read smaller type, that in order to calculate the percentage, 500 cases were specially examined.

A total of 71 per cent. read $6 / 4,6 / 4$, which was the smallest type available.

* Received for publication, March 13, 1946. 


\begin{tabular}{|c|c|c|c|c|c|}
\hline $6 / 6$ & $6 / 6$ & 20 & $4^{\circ} 0$ per & $\mathrm{ce}$ & pprox \\
\hline $6 / 6$ & $6 / 5$ & 18 & 3.5 & , & ", \\
\hline $6 / 6$ & $6 / 4$ & 8 & 1.5 & , & ", \\
\hline $6 / 5$ & $6 / 5$ & 58 & $12^{\circ} 0$ & $"$ & ," \\
\hline $6 / 5$ & $6 / 4$ & 39 & $8^{\circ} 0$ & ", &, \\
\hline $6 / 4$ & $6 / 4$ & 357 & $71^{\circ} 0$ & , & , \\
\hline
\end{tabular}

FIG. 1.

An American Medical Mission visiting the Board in 1941 stated that the average acuity of the fit young men of the U.S.A. was found to be nearer $20 / 15(6 / 4.5)$ than $20 / 20(6 / 6)$. (It is interesting to observe that the Americans, who use the metric system for most scientific measurements, still use English measures in optometry, while the contrary is used in this country).

\section{Manifest hypermetropia}

Many candidates have expressed dissatisfaction after having been assessed as "Unfit Air Crew (EYES)", when they know they have "always read down to the bottom line", and some have returned to ask what was wrong. It is not easy to convince them without divulging the principles of the examination, and although the test is described in ophthalmic text-books, it becomes useless when a candidate who wishes to pass, knows beforehand exactly what he is expected to see.

Some subjects are not troubled by even considerable amounts of hypermetropia but under such circumstances as overwork, worry, and strain, small amounts may produce considerable symtoms of asthenopia.

Pilots with more than $2.5 \mathrm{D}$. of manifest hypermetropia have Hown over 1,000 hours without complaint and one of them had flown without trouble over 2,500 hours with 5 dioptres. Such men might be compared with another who had 1.25 dioptres. Following a period of considerable overwork his punctum proximum receded from $14 \mathrm{cms}$. to $22 \mathrm{cms}$. and he manifested a 2.5 dioptres of hypermetropia with marked symptoms including diplopia. This officer was no longer flying but spent two hours each week on a Link trainer and he soon noticed the difference with his eyes, especially when under the hood. Here the effort of accommodating for the instrument panel at approximately $2 / 3$ metre without relief produced such intense strain that he invariably developed diplopia and fell asleep, the alteration in flying altitude immediately waking him with a start.

The feeling of utter exhaustion could be realised, perhaps, only by one so afflicted, but the danger to a pilot returning from a long and hazardous trip is demonstrated in no uncertain manner. 
On several occasions pilots were examined after two tours of operations, and it was found some of them developed symptoms of asthenopia with approximately only one dioptre of manifest hypermetropia.

Out of a total of 21,622 candidates examined for air crew duties, $478(2 \cdot 21$ per cent.) were rejected for manifest hypermetropia.

\section{The Cover Test}

The cover test is a simple procedure from which a considerable amount of information may be obtained. It is not uncommon to find cases whose near response is a large or moderate deviation with either "slow recovery" or even " no recovery" who are orthophoric for distance with no movement on the cover test. I have found the converse, but the condition is not common.

It would appear an advantage to repeat the cover test for "distance" when it is unsatisfactory for "near" or when there is heterophoria.

I have seen so many cases with unsatisfactory cover tests who were orthophoric for distance, that I wonder whether the desire for fusion may not, perhaps, be occasionally stimulated by the fixation of two such dissimilar objects as the Maddox rod and red light, and thus show no deviation when perhaps a slight imbalance does exist.

For two years, all these cases with doubtful cover tests were examined with the Worth's four dot test which I consider to be of the greatest value. Several cases possessing simultaneous binocular perception would have been placed in a higher category without the results of this test.

\section{The Maddox test}

This test was invariably beyond criticism and a more simple one could hardly be desired.

In a dimly lit room it was noticeable how much difficulty was found by some persons in seeing the red-line and in most cases it was obvious that the ease with which it was seen bore a direct relationship to the man's apparent mental alertness.

Ocular muscle imbalance

\begin{tabular}{|c|c|c|c|}
\hline Total cases & & & 8015 \\
\hline $\mathrm{ESO}$ & $6_{45}^{\Delta}$ to $10^{\Delta}$ & $\underset{34}{\operatorname{over}} 10^{\Delta}$ & $\begin{array}{c}\text { over } 6^{\triangle} \\
\quad 51\end{array}$ \\
\hline EXO & 16 & ó & 43 \\
\hline $\begin{array}{l}\text { Hyper } \quad . . \\
\text { Suppression }\end{array}$ & $1_{5}^{\Delta}$ to $2^{\Delta}$ & $\begin{array}{c}\text { over } 2^{\triangle} \\
31 \text { cases }\end{array}$ & $\underset{37}{\text { over }} 1^{\triangle}$ \\
\hline
\end{tabular}

FIG. 2. 
Strabismus. (Among 22,876 cases). (i) Internal 44: Internal alternating 10. (ii) External 26. External alternating 9. (iii) Vertical 25.

Other muscle abnormalities. (iv). Overaction. Right inferior oblique 2. Left inferior oblique with paresis of right superior rectus 1. (v) Paresis of individual muscle. Left inferior oblique 2. Third nerve (post meningitis) 1. Third nerve 1. Left superior rectus 1. (vi) Limitation. Congenital limitation of all ocular movements 3.

Torticollis. 43 cases were seen, three of whom had facial asymmetry, three vertical squint, and one, ptosis.

In a series of 1,711 cases 52 cases were seen who had one eye higher than the other (27 cases right eye: 25 cases left eye) without either hyperphoria or torticollis.

It is worthy of note that the majority of candidates with an ocular torticollis had no demonstrable muscular imbalance although their eyes were of irregular height. In all cases the head was turned to elevate the higher eye.

Six cases of torticollis were seen which were not ocular in origin.

Restriction of movement. Three cases were seen with a marked limitation of all eye movements.

Ptosis. Eleven cases were seen with unilateral ptosis; Three cases were seen with bilateral ptosis. One with amblyopia. One with well marked arcus (age 17). One with facial palsy.

Nystagmus. Primary. Horizontal 23. Vertical 1. Unilateral 1. SECONDARY 4.

Diplopia was found in four cases. One following ethmoid operation. One following ophthalmoplegia. One with choroidal dystrophy. Two with signs of disseminated sclerosis.

65 cases of amblyopia were recorded who had no muscular imbalance.

\section{Bishop Harman test}

The interpupillary distance was found to vary between $55 \mathrm{mms}$. and $75 \mathrm{~mm}$., the average being about $64 \mathrm{~mm}$.

The phorometer estimates the "desire for binocular vision" and is a dynamic and not a static test like the Maddox wing test.

The estimation is of considerable importance in cases of heterophoria and those showing an abnormal cover test. -

(i) In candidates with weak convergence who are orthophoric for distance, exophoria of 5 to 10 is not unusual.

(ii) Many cases with poor convergence show an outward deviation of one eye (more frequently the left) and a monocular response anywhere between 20 and zero. This signifies a low amplitude of convergence with marked dominance of one eye, a fact usually shown by the-response to the cover test. 


\section{Convergence}

Before 1942, candidates whose convergence was between 10 and . $20 \mathrm{~cm}$. were given orthoptic training and out of 4,231 men examined 120 were sent for that treatment. The convergence of less than $20 \mathrm{~cm}$. caused rejection for pilot training into which category twenty-nine were placed.

It was common to find candidates unable to converge more than $40 \mathrm{~cm}$. who would upon repeated attempts gradually improve. It was not unusual to have one or two in the waiting room practising simple convergence exercises who were, after an hour or so, able to pass the required standard.

Convergence when once developed.appears to remain indefinitely and I have records of one patient who, in 1925, was trained to overcome 65 prism dioptres, and 20 years later, was able to converge 60 degrees on the synoptophore.

\begin{tabular}{cc|c|c|c}
\hline Total Cases & \multicolumn{2}{|c|}{4231} & 8015 \\
\hline Convergençe & $\ldots$ & $10 \mathrm{~cm}$. to $20 \mathrm{~cm}$. & Over $20 \mathrm{~cm}$. & $\begin{array}{c}\text { Over } 10 \mathrm{~cm} . \\
\end{array}$ \\
\hline $\begin{aligned} \text { Approximate } \\
\text { percentage }\end{aligned}$ & 120 & 29 & 53 \\
\hline
\end{tabular}

FIG. 3.

Accommodation

The method of estimating the punctum proximum by approaching a test card with the letters A L T (Jaeger 14) and asking when they become "blurred" or "no longer distinct" can only be considered an approximation.

Even an experienced observer finds some difficulty in deciding precisely when a letter is first blurred or knowing what degree of blurring is expected, and some candidates wait until the letters can no longer be deciphered.

It would appear more accurate to withdraw slowly from the eye (corrected if necessary), a Jaeger I test type, and to note the distance at which it can first be read.

\section{Visual fields}

The value of determining the size of the visual fields by hand movements as a routine is small. This was done in 5,000 cases wtihout finding any defects. 
The test is considered to be too inadequate to demonstrate field changes of which the subject is unaware.

All cases with a history of trauma were examined with the perimeter and four defects were found.

A similar examination was performed in those pathological conditions which indicated it.

\section{Colour vision}

The colour vision of every candidate was tested with the Ishihara or the pseudo-isochromatic plates printed by the American Optical Company. Every candidate who made any error was examined with the Giles-Archer Colour Perception Unit on which his assessment was made.

A total of 40,295 men were seen, out of whom 1,152 ( 3 per cent.) were anomalous trichomats (colour defective safe), and 1,083 (2.5 per cent.) dichromats (colour defective unsafe).

An investigation of the defectives forms the substance of another paper.

A considerable number of patients were re-examined who had been rejected due to persistent errors such as landing "too high" or "too low" during their initial flying training, and several of these were found to have phorias. No records were kept.

On the other hand during the re-examination of experienced pilots for initial " $B$ " licences and the renewal of " $B$ " licences a number were found who were unfit to fly according to the standards and the results of 33 are given in detail.

An analysis shows the following defects:-Manifest hypermetropia 9 ; defective visual acuity 8 ; monocular for near vision 5 ; amblyopic in one eye 2; unsafe co'our vision 2; defective convergence 2 ; over-action of inferior oblique 2 ; external strabismus 1; alternating external strabismus 1; excessive exophoria 1. Total 33.

\section{Fundi and media}

All pathological conditions and congenital abnormalities were classified : 127 fundus drawings were made.

Permission was granted by Sir Harold Whittingham, D.G.M.S., R.A.F., for the publication of these excerpts from the report made at the end of the war on ophthalmic work in the Royal Air Force Volunteer Reserve. 